International Journal of Electrical Engineering and Technology (IJEET)

Volume 11, Issue 4, June 2020, pp. 204-216, Article ID: IJEET_11_04_023

Available online at https://iaeme.com/Home/issue/IJEET? Volume $=11 \&$ Issue $=4$

ISSN Print: 0976-6545 and ISSN Online: 0976-6553

DOI: https://doi.org/10.34218/IJEET.11.4.2020.023

(C) IAEME Publication

Scopus Indexed

\title{
NOVEL ECONOMICAL SOCIAL DISTANCING SMART DEVICE FOR COVID19
}

\author{
Rahul Reddy Nadikattu \\ Department of Information Technology, \\ University of the Cumberlands, United States \\ Sikender Mohsienuddin Mohammad \\ Department of Information Technology, \\ Wilmington University, United States \\ Pawan Whig \\ Dean Research in IT, \\ Vivekananda Institute of Professional Studies, Delhi, India
}

\begin{abstract}
Spiritual intelligence is the science of human energy management that clarifies and in the era of COVID -19 in which everywhere there is a panic like situation and according to the World Health Organization Social Distancing will be proven to be the only solution. In this research paper, an innovative localization method was proposing to track humans' position in an outdoor environment based on sensors is proposed. With the help of artificial intelligence, this novel smart device is handy for maintaining a social distancing as well as detecting COVID 19 symptom patients and thereby safety. In these COVID-19 environments, where everyone is conscious about their safety, we came up with the idea of this novel device. Most of the time, people on the roadside watched their front but were not able to look after what is going on behind them. The device will give alert to the person if someone in the critical range of six feet around him. The method is reasonably accurate and can be very useful in maintaining social distancing. The sensor model used is described, and the expected errors in distance estimates are analyzed and modeled. Finally, the experimental results are presented.
\end{abstract}

Key words: Sensor, WHO, Social Distancing, Tracking, COVID-19, Machine Learning, Python, Smart Device, Artificial Intelligence, Economical.

Cite this Article: Rahul Reddy Nadikattu, Sikender Mohsienuddin Mohammad and Pawan Whig, Novel Economical Social Distancing Smart Device for Covid19. International Journal of Electrical Engineering and Technology, 11(4), 2020, pp. 204-216.

https://iaeme.com/Home/issue/IJEET?Volume=11\&Issue $=4$ 


\section{INTRODUCTION}

Intelligence Since COVID-19 spreads from individual to individual, diminishing the manners in which individuals come in close contact with one another is fundamental. Social separating implies staying at home, however much as reasonably expected, and maintaining a strategic distance. From swarmed, open spots where close contact with others is likely, this is why remain at home requests are set up in such vast numbers of networks, dropping occasions and social affairs of more than ten individuals and shutting shops and cafés, and bars. Its likewise why numerous schools have moved to web-based learning. For essential excursions like shopping for food, the CDC suggests wearing a fabric face covering and remaining in any event 6 feet from others.

Humane removal is a primary method to hinder the spread of COVID-19. Also, it's significant that you follow the common removing suggestions in your locale, regardless of whether you're in one of the high-hazard gatherings or not.

It may with schools shut and individuals telecommuting, it might be enticing to get kids together for playdates or sleepovers or to feel that social occasions of more than ten individuals are protected. In any case, social separating possibly works if we as a whole partake. Also, easing back down or forestalling the spread of the infection will spare lives.

The spread of COVID-19 has been the fast and bureaucratic, state, and neighborhood governments are doing whatever is essential to shield we all from becoming ill. While many people who become tainted will have indications like cold or seasonal influenza, and youngsters appear to be less influenced by the infection than grown-ups, we are liable for ensuring those at higher hazard. Steps like social removing may feel like a burden. However, it's the ideal way right presently to secure our family, companions, and neighbors who might be helpless.

A coronavirus is a kind of typical infection that taints our upper respiratory tract framework, including throat and nose. It gets the name from its crown-like shape when watched under a magnifying instrument. According to the World Health Organization (WHO), pneumonia of obscure reason identified in Wuhan, China, was first answered to the WHO Country Office in China on 31st December 2019. The episode was proclaimed a Public Wellbeing Emergency of International Concern on 30th January 2020. On eleventh February 2020, WHO reported a name for the new coronavirus ailment: COVID-19. The circumstance was observed intently alongside day by day appraisal of the number of affirmed instances of COVID-19 over the globe. According to the WHO report, in the previous fourteen days, the name of the cases of COVID-19 outside China had expanded 13-crease, and the quantity of influenced nations significantly increased. With more than 1,18,000 cases in 114 countries and 4,291 individuals who have lost their lives, COVID-19 was spreading alarmingly.

In the resulting days and weeks ahead, it expected that the number of cases, the number of passing's, and the quantity of influenced nations would increment further. Therefore, the WHO described COVID-19 as a pandemic [1]. It spreads through the novel Corona Virus. The microscopic picture of Coronavirus appears in Fig. 1. 


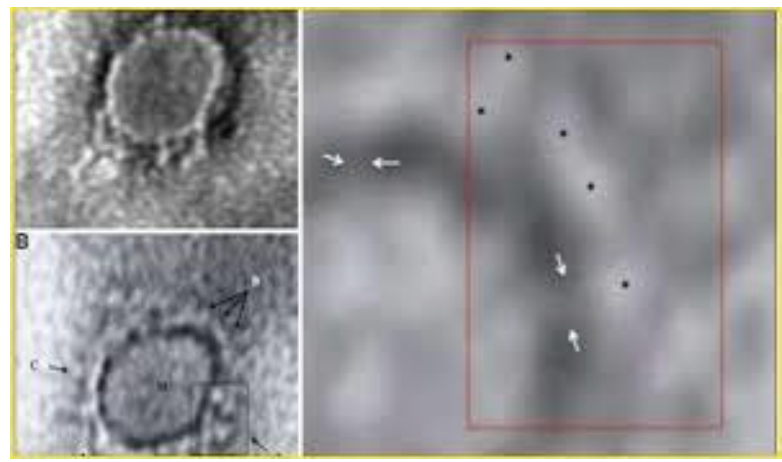

Figure 1 Visual PLS estimates of structural model co-efficient for the model explaining the relationship among spiritual intelligence, task and contextual performance

The number of people been infected with this deadly disease is increasing in India $\&$ the USA also. WHO has taken active steps intending to curb the spread of this disease. As per an advisory issued the suspected patient should look for the following signs and symptoms:

Fever

Cough

Shortness of breath or difficulty in breathing

Presently there is no medicine to cure or vaccine to prevent COVID-19. As they say, prevention is better than cure, is aptly suitable in the current scenario. The best ways to avoid infection is to get exposure to the virus through the following measures:

Observe good personal Hygiene

Practice frequent hand washing with soap

Covering mouth when coughing and sneezing

Social Distancing

As per Health Experts, Social Distancing can be an effective measure to curtail the spread of COVID-19. Social distancing is purposely maintaining the physical space between individuals to prevent the spread of illness. Keeping some distance of at least one meter from other people lessens the chances of getting infected with COVID-19. Social distancing is a prevention and control intervention implemented to decrease contact between those infected with a disease-causing pathogen and to slow down the rate and extent of disease transmission in a community. For the practical impact of social distancing, the authorities have also taken the following steps:

Work from home

Closing of Educational Institutions and Coaching centers and shifting to online mode

Maintaining in touch with near and dear ones using audio and video calls

Annulling or deferring meetings, seminars and conferences.

There may be instances where people's movement or traveling is necessary to provide essential services like food, medicine, hospitals, banks, etc. Social distancing in such scenario can be in the following manner:

Keeping a distance of at least 1-1.5 meter between individuals

Greet people with Namaste instead of handshakes

Usage of electronic money instead of currency

Avoid public transport

for shopping

Maintaining a distance of at least 1-1.5 meter while standing in a queue 
The individuals who are staying at home should take the following precautions:

Wash hands frequently with soap

Request visitors to avoid coming home

Avoid visiting markets and shops for buying essentials and prefer the home delivery option

Frequently sanitize surfaces like door knobs, taps, kitchen surfaces and other objects that are touched on a regular basis

Technology will effectively be used for maintaining the requisite distance as per social distancing norms. The proposed system uses a wearable device capable of sensing the distance between two individuals and triggering an alarm in the event of proximity between the individuals.

\section{BLOCK DIAGRAM OF SYSTEM}

The wearable device consists of PIR Sensor, micro-controller, and mobile for display and gives alert to the user. The sensor will detect the distance between the wearer and other individuals. The micro-controller is programmed to verify the desired length and trigger the alarm as well as give the mobile alert to the user. The proposed system is shown in Fig. 2.

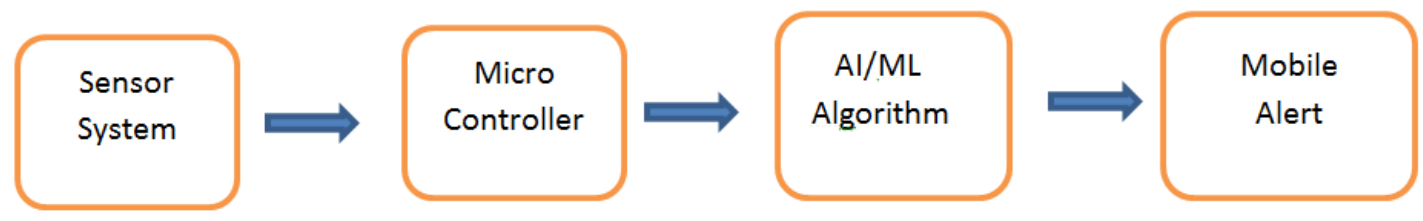

Figure 2 Block diagram of the proposed system

Description of hardware components used are as Follow

\subsection{Sensor System}

PIR Sensor is also called Passive Infrared Sensors. These sensors are used to detect human movement when it comes in a particular range. The range of coverage depends upon the type and design of Sensor. The PIR sensor is shown in Fig. 3

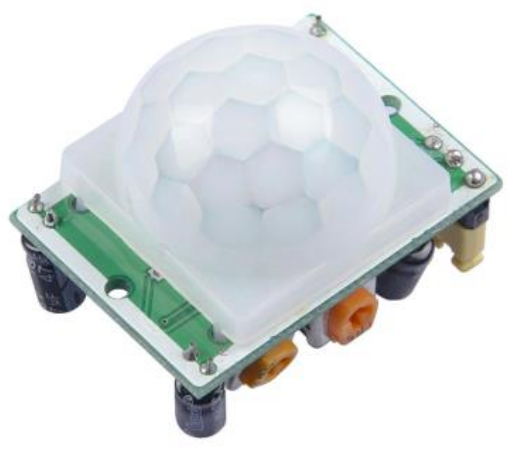

Figure 3 Structure of PIR Sensor

The PIR sensor does not radiate energy to space on the other hand its working relies on receiving infrared radiation from the human body to make an alert into mobile device. The temperature of the normal human body is 36-27 ${ }^{\circ} \mathrm{C}$, and most of its radiant energy concentrated in the wavelength range of 8-12 $\mu \mathrm{m}$. If the human infrared radiation directly irradiated on the radar, it will cause a temperature change to output a signal. Still, in doing so, the detection distance will not be far. To lengthen the detector's detection distance, an optical 
system must be added to collect the infrared radiation, using a flexible visual reflection system or a Fresnel lens, which is made of plastic as a focusing system for IR radiation.

Technology In the location territory, the infrared radiation vitality of the human body through the attire got by the focal point of the indicator and spotlight on the pyroelectric sensor. The human body (gatecrasher) will move in this observation mode; it moves to a specific field of view and leaves the field of view. The pyroelectric sensor sees the moving human body for some time and afterward doesn't see it, so the human body. The IR radiation continually changes the pyroelectric material's temperature with the goal that it yields a relating signal, which is the alert sign.

The various features of different types of PIR Sensors with their angle of operation and the direction ranges are given in Table 1

Table 1 Features of Different types of PIR Sensor

\begin{tabular}{|c|c|c|}
\hline Product & $\begin{array}{l}\text { Measuring } \\
\text { range (theoretical } \\
\text { value) }\end{array}$ & Features \\
\hline $\begin{array}{l}\text { Grove - PIR Motion } \\
\text { Sensor }\end{array}$ & $\begin{array}{l}\text { Max Distance:3- } \\
6 \mathrm{~m}(3 \mathrm{~m} \text { by } \\
\text { default }) \quad \text { Angle:< } \\
120^{\circ}\end{array}$ & $\begin{array}{l}\text { Measuring distance and holding } \\
\text { time adjustable response } \\
\text { speed: } 0.3 s-25 s\end{array}$ \\
\hline $\begin{array}{l}\text { Grove-Adjustable PIR } \\
\text { Motion Sensor }\end{array}$ & $\begin{array}{l}\text { Max Distance: } 3- \\
6 \mathrm{~m} \quad \text { Angle: } X=110^{\circ} \\
Y=90^{\circ}\end{array}$ & $\begin{array}{l}\text { Measuring distance and holding } \\
\text { time adjustable }\end{array}$ \\
\hline $\begin{array}{l}\text { Grove - mini PIR motion } \\
\text { sensor }\end{array}$ & $\begin{array}{l}\text { Max Distance: } 2- \\
5 \mathrm{~m}(2 \mathrm{~m} \text { by } \\
\text { default }) \quad \text { Angle: } \mathrm{X}=11 \\
0^{\circ} \mathrm{Y}=90^{\circ}\end{array}$ & Sensitivity adjustable Mini-size \\
\hline $\begin{array}{l}\text { PIR Motion Sensor - } \\
\text { Large Lens version }\end{array}$ & $\begin{array}{l}\text { Max } \\
\text { Distance:9m Angle: } \\
<120^{\circ}\end{array}$ & $\begin{array}{l}\text { Large lens which can support long } \\
\text { range and wide angle } 2.54 \mathrm{~mm} \\
\text { standard connector }\end{array}$ \\
\hline $\begin{array}{l}\text { PIR Motion sensor } \\
\text { module }\end{array}$ & $\begin{array}{l}\text { Max Distance:3- } \\
7 \mathrm{~m} \quad \text { Angle }<120^{\circ}\end{array}$ & Measuring distance adjustable \\
\hline $\begin{array}{l}\text { Mini PIR Motion Sensor } \\
\text { Module }\end{array}$ & $\begin{array}{l}\text { Max } \\
\text { Distance:7m Angle: } \\
<100^{\circ}\end{array}$ & Mini-size \\
\hline
\end{tabular}




\subsection{Microcontroller}

There is various micro-controller available like aurdino and raspberry pie. The architecture of Aurdino given below with the pin description. The simulation of the device was done using the Arduino micro-controller, and the actual design was realized using raspberry pie.

Table 2

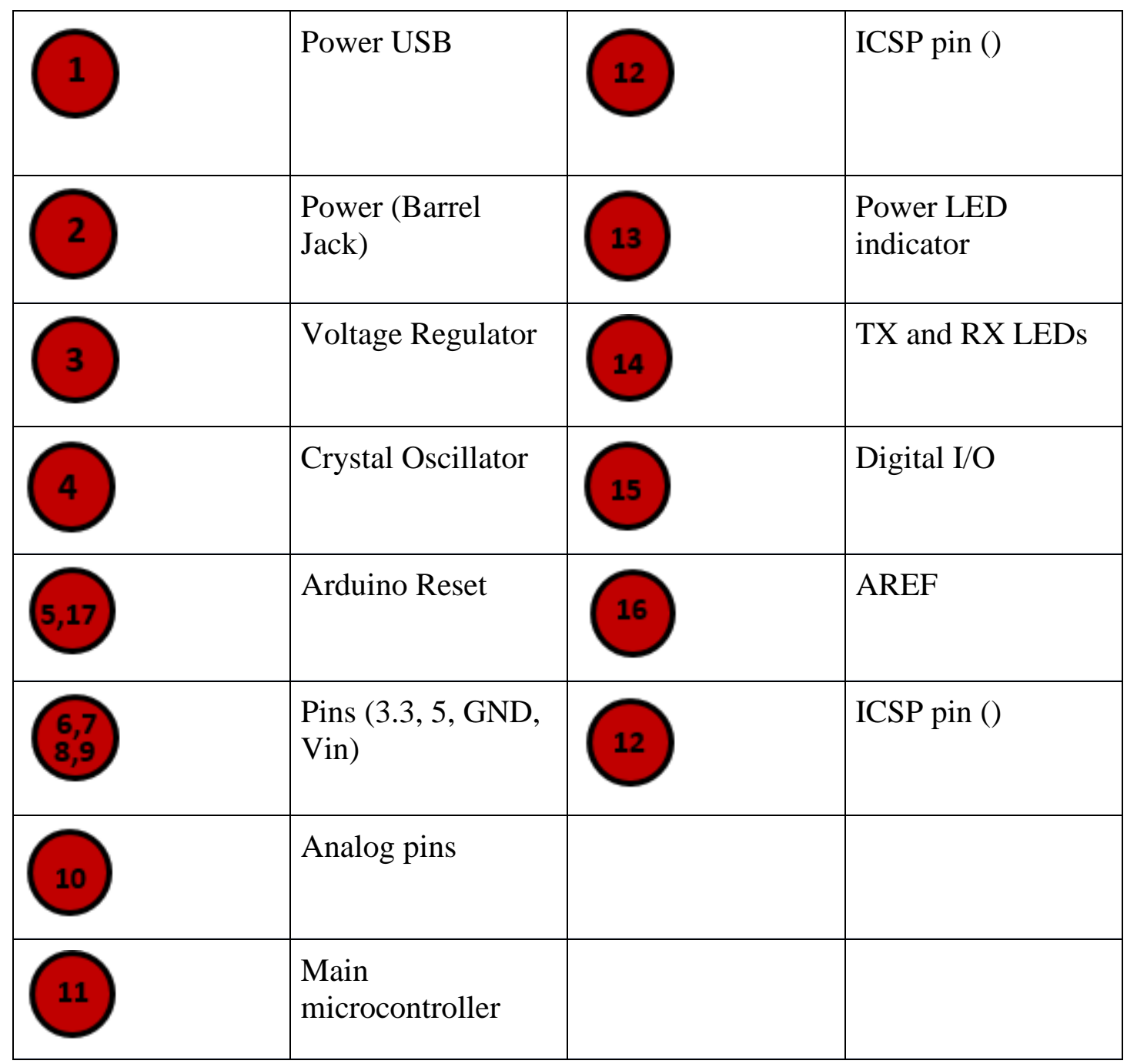

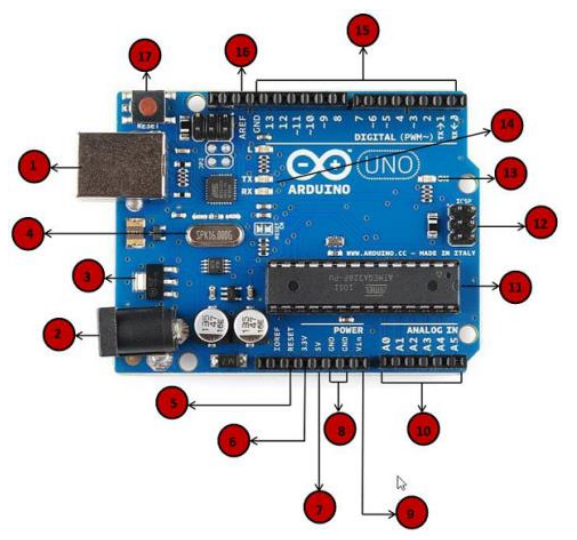

Figure 4 Architecture of Arduino board 


\section{WORKING OF DEVICE}

The proposed device based on the principle of a PIR sensor on receiving infrared radiation from the human body to make an alarm and alert on the mobile device. Also, calibrating the infrared radiation received from the sensor into the thermal conductivity temperature of the body can be calculated. This way, the device will measure not only social distancing but also the heat of the person in the range. Using a Machine learning algorithm, it detected that the person has COVID -19 symptoms or not. Any object with temperature is always radiating infrared rays to the outside world. Usually, the surface temperature of the human body is 36$27^{\circ} \mathrm{C}$, and most of its radiant energy concentrated in the wavelength range of 8-12 $\mu \mathrm{m}$. In this Novel Device, a pair of PIR sensors are used, which will cover almost $240^{\circ}$ around the wearable, and based on radiating infrared rays, it will produce an alert to the wearable. The Extension of this device using artificial intelligence and machine learning also alerts the possibility of corona patients around you. The total angle covered around the wearable shown in Fig. Five, it indicates the device will include $240^{\circ}$ around the wearable.

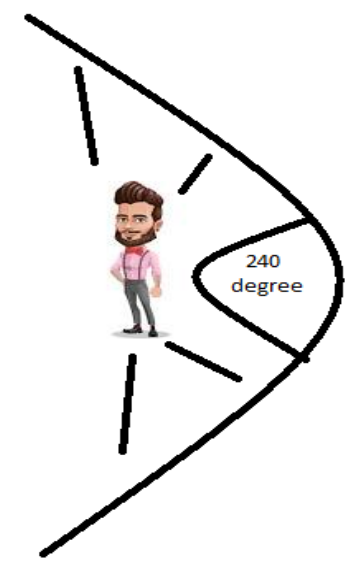

Figure 5 Angle covered around wearable

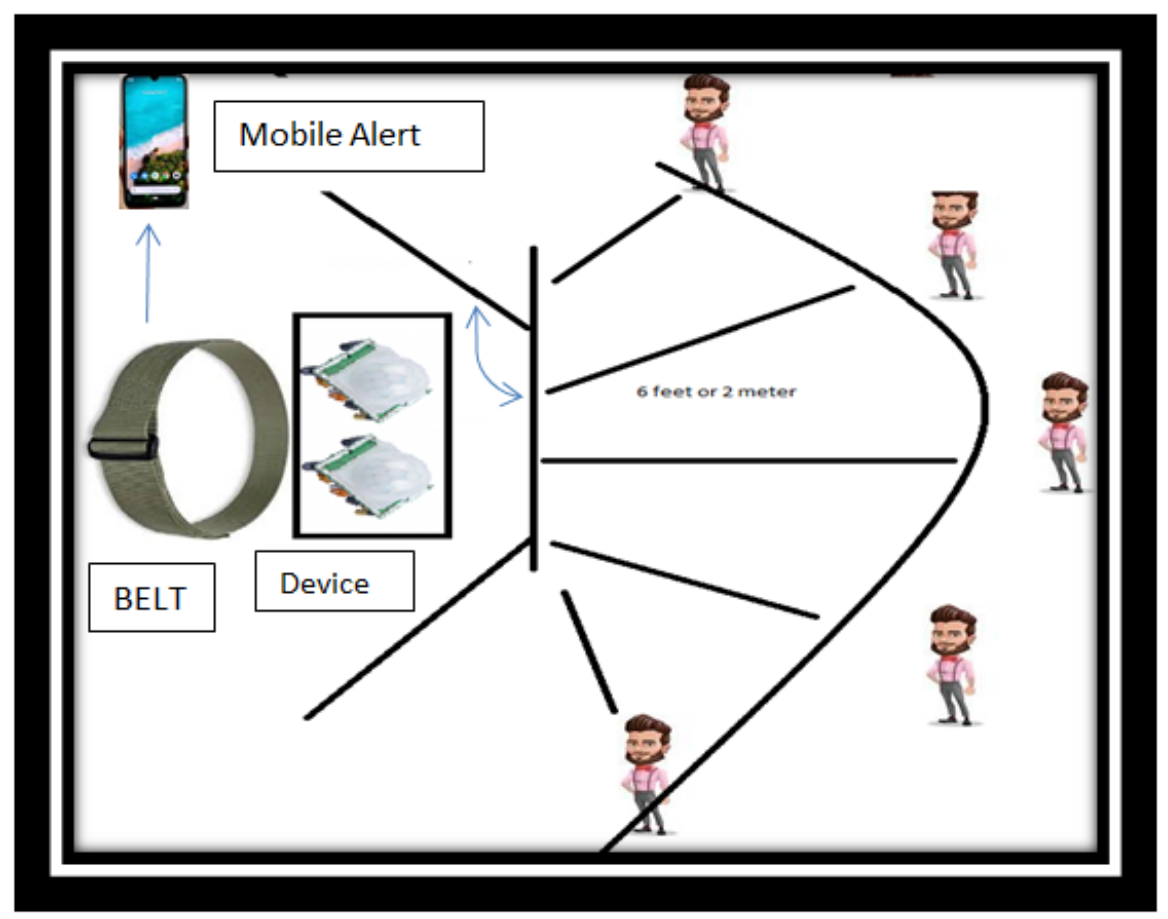

Figure 6 Working Diagram of System 
The working Diagram of the device shown in Fig. 6. It depicts that when someone comes in the critical range of social distancing, then an alert has been initiated in the mobile of the person and then using ML algorithm advice has been issues that "Someone behind you" also based on the temperature of the body the probability of symptoms of COVID calculated.

\section{MATHEMATICAL MODELING}

The device follows photometry inverse law. The simple equation for measuring distance $\mathrm{x}$ and the angle of incidence $\theta$ with target surface

$\mathrm{S}(\mathrm{x}, \theta)=\frac{\alpha}{x^{2}} \cos \theta+\beta$

Where,

$\mathrm{S}(\mathrm{x}, \theta)$ is the sensor output, $\mathrm{x}$ is distance and

$\theta$ is the angle of incidence with target surface.

$\alpha$ and $\beta$ are the sensor model parameters

$\alpha$ depends upon radiant intensity of material of sensor, spectral sensitivity of photodiode used, gain of amplifier and reflectivity coefficient of target.

$\beta$ is the parameter which depends upon amplifier offset plus ambient light effect to minimize the effect of $\beta$ equation 1 can be written as

$\mathrm{y}(\mathrm{x}, \theta)=\mathrm{S}(\mathrm{x}, \theta)=\stackrel{-\beta}{\frac{\alpha}{x^{2}} \cos \theta}$

using above equation the distance of the person can be calculated as shown in figure 5 .

From equation 2

$\mathrm{X}=\sqrt{\frac{\alpha}{y} \cos \theta}=\sqrt{\frac{\alpha}{y}} \sqrt{\cos \theta}$

Let two PIR sensor measuring flat surface with an angle of incidence $\theta$ and value of $y_{1}$ and $y_{2}$ can be calculated if value of $\theta=0$

$x_{1}^{\prime}=\sqrt{\frac{\alpha}{y_{1}}} \quad x_{2}^{\prime} \sqrt{\frac{\alpha}{y_{2}}}$

Using snells law the ratio of two incident angle is calculated as

Let $\mathrm{R}^{\prime}=\tan \theta^{\prime}$ and $\mathrm{R}=\tan \theta$

Where $\theta$ is the actual angle of incidence and $\theta^{\prime}$ is the apparent angle of incidence The relationship between $\mathrm{R}$ and $\mathrm{R}^{\prime}$ is

$\mathrm{R}=$

$$
R^{\prime} \sqrt{\cos \theta}
$$

And hence to obtain $\theta$ the following equation must be solved

$R^{\prime}(\cos \theta)^{3}+(\cos \theta)^{2}-1=0$ 
With the different value of $\theta$ taking from range -120 to 120 and $\mathbf{R}^{\prime}$ a line is fitted with a coefficient of correlation 0.95 and having standard error of 0.583019 by considering the typical values of $\alpha=0.7$ and $\beta=0.01$. the graph between the sensor output and with distance is shown in Fig. 7

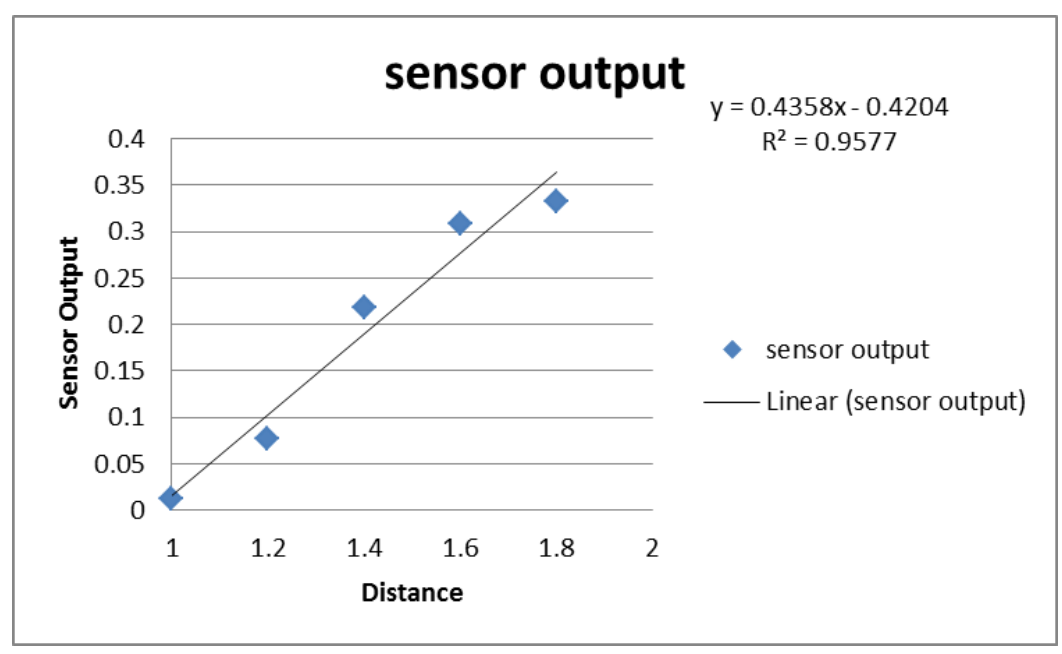

Figure 7 Graph between sensor output and distance

\subsection{Normal Probability Plot}

There is a specific case for the likelihood plot, which is known as the standard likelihood plot. It is a graphical technique used to check the ordinary appropriation of the information. On plotting the focuses, a roughly straight line framed, which shows that typical circulation is a nice model for a given arrangement of information of sensor yield and the separation of the individual. The standard likelihood plot for the framework is plotted and appeared in Fig. 8

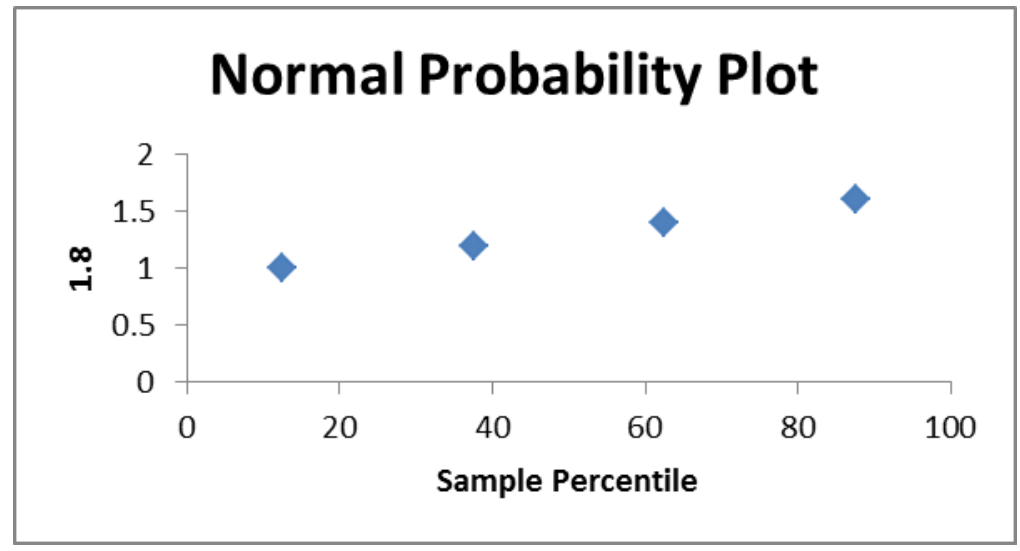

Figure 8 Normal Probability for the sensor

\subsection{Residual Plot}

It is also called lingering plot. A lingering plot is a plot between a yield and information factors used to show whether the given relapse model is proper as indicated by your information. There ought not be any unmistakable example for the plotted residuals, and they should be arbitrary. Great relapse models by and large get uncorrelated residuals. The remaining plot for the created framework has arranged and appeared in Fig 9. 


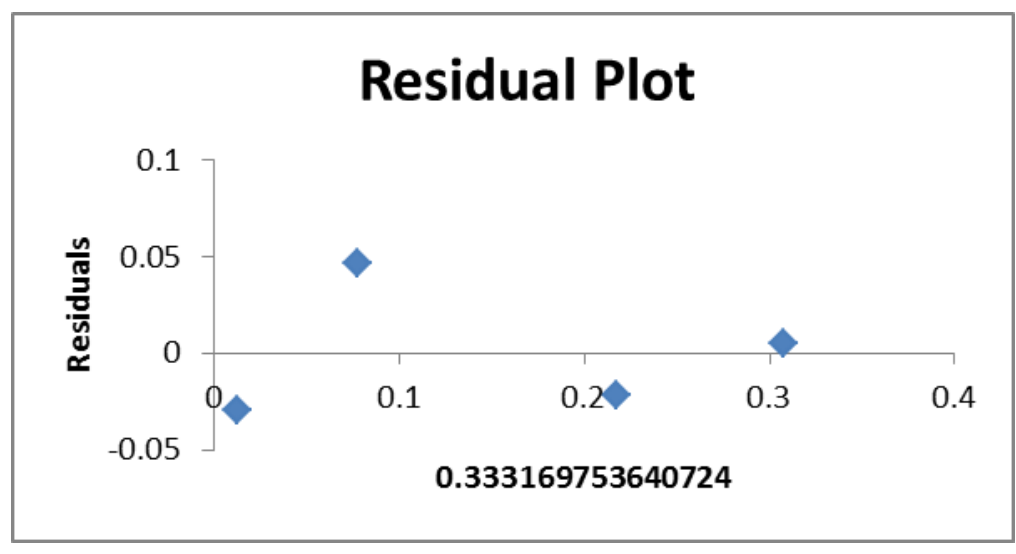

Figure 9 Residual plot for the device

In the relapse measurements of a gadget has been done and appeared in Table2. The table incorporates various estimations of numerous $\mathrm{R}, \mathrm{R}$ square, and balanced $\mathrm{R}$ square, the standard blunder as acquired from the examination. The measure of $\mathrm{R}$ Square shows the straight relationship of one boundary concerning others. The Value of R-Square is exceptionally near solidarity demonstrates that the extent of the change of one variable is unsurprising from the other variable.

The standard mistake is the distinction in the estimation of the genuine and assessed variable. The least the measure of standard mistake shows how close the genuine and anticipated worth is or the gadget's affectability. The expense of the standard blunder is little, which shows a superior precision of the gear.

Table 2 Regression statistics

\begin{tabular}{|l|l|}
\hline \multicolumn{2}{|c|}{ Regression Statistics } \\
\hline Multiple R & 0.990993 \\
\hline R Square & 0.982068 \\
\hline Adjusted R Square & 0.973102 \\
\hline Standard Error & 0.042346 \\
\hline Observations & 14 \\
\hline
\end{tabular}

\section{Inference from Table 2}

(a) The value of $\mathrm{R}^{2}$ in the case of a sensor model which shows the direction of a linear relationship between the distance between the persons and sensor output.

(b) The value of standard error found to be smaller, which shows better accuracy of the model.

The ANOVA analysis for the device presented in Table 2

ANOVA examination is a straightforward investigation of change on information for at least two factors. The ANOVA table gives the accompanying data:

Degrees of opportunity (df), The Sum of the Squares (SS), The Mean Square (MS), The F proportion (F), The Significance $\mathrm{S}$.

ANOVA examination is basic to test the theory for each example esteem with a similar likelihood dissemination. A high $\mathrm{F}$ esteem speaks to there is an insignificant deviation between the methods. The measure of ways is the equivalent, which prompts minimal estimation of standard blunder, and the gadget is sensibly precise. 
Table 3 ANOVA Analysis

\begin{tabular}{lccccc}
\multicolumn{1}{c}{ ANOVA } & \multicolumn{1}{c}{} & & $\begin{array}{c}\text { Significanc } \\
\boldsymbol{e} \boldsymbol{F}\end{array}$ \\
\hline Regression & $\boldsymbol{d} \boldsymbol{f}$ & $\boldsymbol{S S}$ & $\boldsymbol{M S}$ & $\boldsymbol{F}$ & 0.009007 \\
Residual & 1 & 0.196414 & 0.196414 & 109.531 & 0.000 \\
Total & 2 & 0.003586 & 0.001793 & & \\
\hline
\end{tabular}

\section{DEVICE SIMULATION AND RESULT}

The hardware implementation for the social distance monitoring system is shown in Fig. 10. The simulation of the device has done, and the results found to be within range. The real-time simulation of the device using a micro-controller and sensor is shown in Fig. 11. It is shown that when someone comes inside the critical field of six feet, the alert will be given on the mobile with the indication that whether a person in the range having COVID symptoms or not. On plotting a trend line between sensor output and distance, the coefficient of determination $\mathrm{R}^{2}$ found to be 0.95 , which is close to 1 . It shows that the regression line is an excellent fit to the data. The standard probability and residual plot for the system are given in Fig. 8 and Fig. 9. Both plots validate that the regression model obtained is appropriate, and thus accuracy of the system is verified. The system has excellent skills and is less complicated. The system is highly suitable for maintaining social distance and detecting COVID symptom patients.

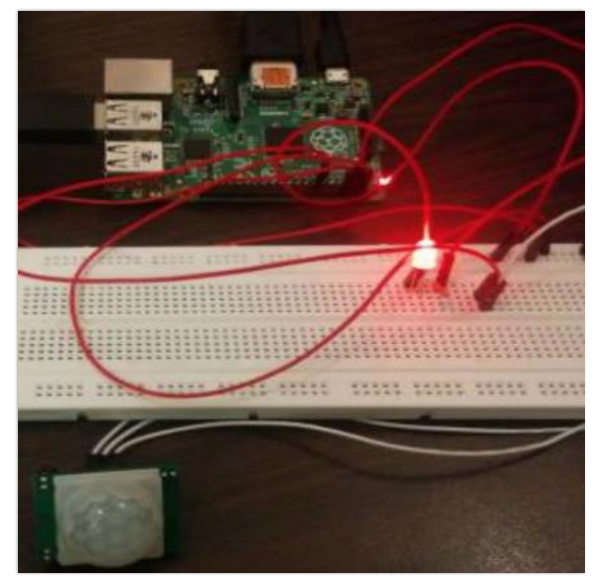

Figure 10 LED gives output when someone in the range of six feet

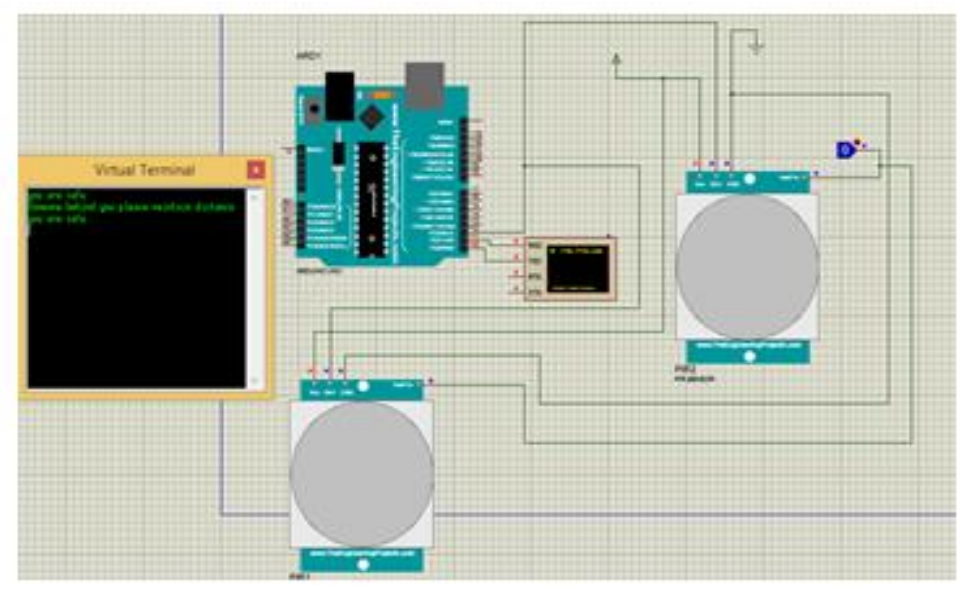

Figure 11 Simulation of the device 


\section{CONCLUSION}

This advantage of this novel device is that it does not only help in maintaining social distancing but also give alert whether person in a range having COVID symptoms or not. The accuracy of the device can be increased by improving the design of the sensor. This research study will help researchers working in the same field.

\section{CONFLICT OF INTEREST}

I hereby, declare that in this research studies there is no conflict of interest

\section{REFERENCES}

[1] R. E. Park, (1924) "The concept of social distance: As applied to the study of racial relations", Journal of applied sociology, vol. 8, pp. 339334.

[2] E. S. Bogardus, (1947) "Measurement of personal-group relations", Sociometry, vol. 10, no. 4 , pp. 306311.

[3] Nadikattu, Rahul Reddy, A Comparative Study between Simulation of Machine Learning and Extreme Learning Techniques on Breast Cancer Diagnosis. Available at SSRN: https://ssrn.com/abstract=3615092

[4] N. Karakayali, (2009) "Social distance and affective orientations 1", Sociological Forum, vol. 24, no. 3, pp. 538562.

[5] M. S. Granovetter, (1977) "The strength of weak ties", Social networks, pp. 347367.

[6] G. Simmel, (1971) On individuality and social forms: Selected writings, University of Chicago Press.

[7] P. Bourdieu, (1989) "Social space and symbolic power", Sociological theory, vol. 7, no. 1, pp. 1425.

[8] Y. Trope, (2012) "Construal level theory", Handbook of theories of social psychology, pp. 118134.

[9] P. Aguilar, S. Brussino and J.-M. Fernandez-Dols, (2013) "Psychological distance increases uncompromising consequentialism", Journal of Experimental Social Psychology, vol. 49, no. 3, pp. 449452.

[10] A. Sardar, M. Joosse, A. Weiss and V. Evers, (2012) "Dont stand so close to me: users attitudinal and behavioral responses to personal space invasion by robots", Proceedings of the seventh annual ACM/IEEE international conference on Human-Robot Interacton., pp. 229230.

[11] Y. Kim, S. S. Kwak and M.-S. Kim, (2013) "Am I acceptable to you? effect of a robots verbal language forms on peoples social distance from robots", Computers in Human Behavior, vol. 29, no. 3, pp. 10911101.

[12] Ajay Rupani, Pawan Whig , Gajendra Sujediya and Piyush Vyas, (2017) "A robust technique for image processing based on interfacing of Raspberry-Pi and FPGA using IoT," International Conference on Computer, Communications and Electronics (Comptelix), IEEE Xplore: 18 August

[13] Jacob B. Chacko; Pawan Whig, (2017) "Low Delay Based Full Adder/Subtractor by MIG and COG Reversible Logic Gate", 2016 8th International Conference on Computational Intelligence and Communication Networks (CICN), IEEE Xplore: 26 October

[14] Purva Agarwal and Pawan Whig, (2017) "Low Delay Based 4 Bit QSD Adder/Subtraction Number System by Reversible Logic Gate", 2016 8th International Conference on Computational Intelligence and Communication Networks (CICN), IEEE Xplore: 26 October

[15] Rahul Reddy Nadikattu, (2020)" Research On Data Science, Data Analytics And Big Data", International Journal of Engineering, Science and Mathematics, Vol. 9, pp.99-105. 
[16] M. Haring, D. Kuchenbrandt and E. Andre, (2014) "Would you like to play with me? how robots group membership and task features influence human robot interaction", 2014 9th ACM/IEEE International Conference on Human-Robot Interaction (HRI), pp. 916.

[17] C. Nass, J. Steuer and E. R. Tauber, (1994) "Computers are social actors", Proceedings of the SIGCHI conference on Human factors in computing systems, pp. 7278.

[18] A. Edwards, C. Edwards, D. Westerman and P. R. Spence, (2019) "Initial expectations interactions and beyond with social robots", Computers in Human Behavior, vol. 90, pp. 308314.

[19] Y. Trope and N. Liberman, (2010) "Construal-level theory of psychological distance", Psychological review, vol. 117, no. 2, pp. 440.

[20] A. Edwards, (2018) "Animals humans and machines: Interactive implications of ontological classification", Human-machine communication: Re-thinking communication technology and ourselves, pp. 2949.

\section{AUTHORS BIOGRAPHY}

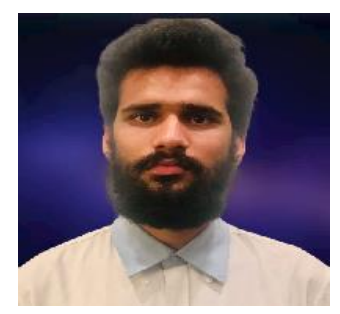

Engineer with more than five years of experience in research and Ph.D. candidate trained in Information Technology with research knowledge and strong communication skills developed from academic and professional experience and ability to work independently or as part of a team. Special expertise in the following IT areas. Serving as Editorial Board Member and Reviewer of Several International Journals. Expertise in Cloud Computing, Artificial Intelligence, Cyber Security, Risk Management in IT, Emerging threats and countermeasures, Information Governance.

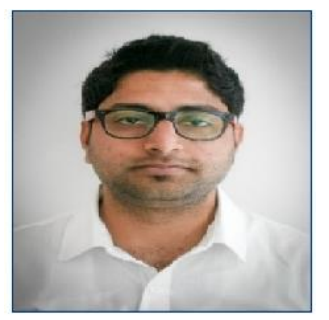

Certified AWS Professional architect. Experienced information technology professional, well-versed in all phases of software development life cycle with emphasis in areas like requirements, development, quality assurance, release, and configuration management. Automation release engineer working with DevOps tools which facilitates collaboration and optimization across the business and technical domains. Using the DevOps lean and agile roots, I have assisted organization to respond faster to changes in various environments. Assisting state and local governments in migrating major workloads, Advancement of legacy systems to implement a full integration pipeline services. My expertise has been around automating the services both on on-premise and on cloud using machine learning, artificial intelligence and software engineering mechanism.

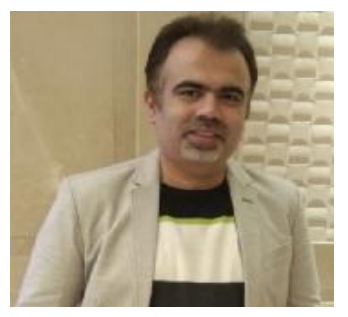

Google Certified in Digital Marketing, having Machine Learning from Stanford University. He is working in the field of IT from last 15 years. He is reviewer of several internationals referred journals. He is member of international association of engineers Hong kong, ISTE, IEEE, IEI and Computer Society of India (CSI). He published technical articles in more than 50 nationals and internationals journals. He has wide area of research like Analog Signal Processing, Sensor Modeling, Water Quality Monitoring Applications and Simulation \& design. Experienced Registrar with a demonstrated history of working in the education management industry. Skilled in Computer-Aided Design (CAD), Curriculum Development, Management Information Systems (MIS), Soft Skills, and Research and Development (R\&D). Strong human resources professional graduated from GBSSS rani bagh. 